PROCEEDINGS OF THE

AMERICAN MATHEMATICAL SOCIETY

Volume 133, Number 8, Pages 2449-2459

S 0002-9939(05)08135-9

Article electronically published on March 21, 2005

\title{
NONNEGATIVELY AND POSITIVELY CURVED INVARIANT METRICS ON CIRCLE BUNDLES
}

\author{
KRISHNAN SHANKAR, KRISTOPHER TAPP, AND WILDERICH TUSCHMANN
}

(Communicated by Wolfgang Ziller)

\begin{abstract}
We derive and study necessary and sufficient conditions for an $S^{1}$-bundle to admit an invariant metric of positive or nonnegative sectional curvature. In case the total space has an invariant metric of nonnegative curvature and the base space is odd dimensional, we prove that the total space contains a flat totally geodesic immersed cylinder. We provide several examples, including a connection metric of nonnegative curvature on the trivial bundle $S^{1} \times S^{3}$ that is not a product metric.
\end{abstract}

\section{INTRODUCTION}

Let $N$ denote the total space of a principal $S^{1}$-bundle over a compact manifold $B$. A metric on $N$ will be called an invariant or a warped connection metric if the principal $S^{1}$-action is by isometries. If in addition all fibers have the same length, then the metric is called a connection metric. If $B$ admits a metric of nonnegative sectional curvature, then it is not known except in special cases whether $N$ must admit an invariant metric or a connection metric of nonnegative sectional curvature. In contrast, the following results are known. If $B$ admits a metric of almost nonnegative curvature, then $N$ admits a connection metric of almost nonnegative curvature [5]. If $B$ has a metric of positive Ricci curvature and $\pi_{1}(N)$ is finite, then $N$ has an invariant metric with positive Ricci curvature for which the projection $N \rightarrow B$ becomes a Riemannian submersion ([6, 8]). Only the trivial principal $S^{1}$-bundle over $\mathrm{T}^{2}$ admits an invariant metric of nonnegative sectional curvature (this follows from [13]).

If $B$ is simply-connected with nonnegative sectional curvature, it is possible that all principal $S^{1}$-bundles over $B$ admit invariant metrics of nonnegative curvature. If $N$ admits an invariant metric with nonnegative sectional curvature, then so does the associated $\mathbf{R}^{2}$-bundle $\left(N \times \mathbf{R}^{2}\right) / S^{1}$, which relates the circle bundle question to the rank 2 case of Cheeger and Gromoll's converse Soul Theorem problem.

We now exhibit some of the known examples. All principal $S^{1}$-bundles over $\mathbf{C P}^{n} \#-\mathbf{C P}^{n}$ admit nonnegative curvature by [17. In [1] Aloff and Wallach found positively curved connection metrics on principal $S^{1}$-bundles over the flag manifold

Received by the editors October 15, 2002 and, in revised form, April 14, 2003.

2000 Mathematics Subject Classification. Primary 53C20.

Key words and phrases. Nonnegative sectional curvature, principal circle bundles, connection metrics.

The first author was supported in part by NSF grant DMS-0103993.

The third author's research was supported by a DFG Heisenberg Fellowship.

(C)2005 American Mathematical Society Reverts to public domain 28 years from publication 
$\mathrm{SU}(3) / \mathrm{T}^{2}$. In [3] (see also [4]) Eschenburg found positively curved invariant metrics on $S^{1}$-bundles over the twisted flag manifold $\mathrm{SU}(3) / / \mathrm{T}^{2}$. It is not known whether these Eschenburg spaces also admit connection metrics of positive (or even nonnegative) curvature, so allowing the fiber-lengths to vary may help realize curvature properties.

The goal of this paper is to derive and study necessary and sufficient conditions under which $N$ admits an invariant metric with nonnegative or positive sectional curvature. We treat nonoriented circle bundles as well. Our conditions generalize Yang's conditions in [17] for $N$ to admit a connection metric of nonnegative curvature.

It is a pleasure to thank J.-H. Eschenburg for illuminating discussions and Wolfgang Ziller for helpful comments. We also wish to thank Burt Totaro for bringing the reference [9] to our attention and the referee for valuable remarks.

\section{Preliminaries}

In order to include nonoriented $S^{1}$-bundles, we work in the following generality:

Definition 2.1. A connection metric on a circle bundle $S^{1} \hookrightarrow N \stackrel{\pi}{\rightarrow} B$ is a metric on $N$ for which $\pi$ is a Riemannian submersion with totally geodesic fibers. A warped connection metric is one obtained from a connection metric by rescaling the fiber metric by a smooth function $f: B \rightarrow \mathbf{R}^{+}$.

For oriented (equivalently, principal) $S^{1}$-bundles, Definition 2.1 agrees with the definition in the Introduction. To see this, first note that if $\pi$ is a Riemannian submersion with totally geodesic fibers, then the $S^{1}$-action on $N$ which rotates the fibers at a common speed sends unit-length vertical (resp. horizontal) vectors to unit-length vertical (resp. horizontal) vectors. The action is therefore by isometries, and remains so after the fibers are rescaled by any smooth function $f: B \rightarrow \mathbf{R}^{+}$. Conversely, if the principal $S^{1}$ action on $N$ is by isometries, then there is an induced metric on $B=N / S^{1}$ for which $\pi: N \rightarrow B$ becomes a Riemannian submersion. If the fibers of this submersion are rescalled to a common length, they become totally geodesic.

The horizontal distribution of a warped connection metric is the kernel of a principal connection form, $\theta$, on the bundle. The curvature form $\Omega=d \theta$ is the pull-back $\Omega=\pi^{*}(\omega)$ of a closed two-form $\omega$ on $B$. The cohomology class of $\omega$ satisfies $[\omega]=2 \pi e$, where $e$ is the real Euler class of the bundle.

In fact, a warped connection metric, $\left(N, g_{N}\right)$, on an oriented circle bundle $S^{1} \hookrightarrow$ $N \stackrel{\pi}{\rightarrow} B$ is determined by choosing (i) a metric $g_{B}$ on $B$, (ii) a principal connection $\theta$ on the bundle, and (iii) a smooth function $f: B \rightarrow \mathbf{R}^{+}$. Then there is a unique metric $g_{N}$ on $N$ such that $\pi:\left(N, g_{N}\right) \rightarrow\left(B, g_{B}\right)$ is a Riemannian submersion whose horizontal space equals the kernel of $\theta$, such that the circumference of each fiber $\pi^{-1}(p)$ equals $2 \pi \cdot f(p)$, and the principal $S^{1}$ action on $\left(N, g_{N}\right)$ is by isometries. In this way we define the warped connection metric determined by $\left\{g_{B}, \theta, f\right\}$. When $f$ is a constant function, $g_{N}$ is a connection metric.

The warped connection metric determined by $\left\{g_{B}, \theta, f\right\}$ makes sense for nonorientable bundles as well, as long as $\theta$ is interpreted as a connection in the associated $\mathbf{R}^{2}$ bundle compatible with a fixed Euclidean structure. Then $\theta$ induces a distribution, $\mathcal{H}$, on $N$, thought of as the bundle of unit-length vectors. Furthermore, there is a unique metric, $g_{N}$, on $N$ such that $\pi:\left(N, g_{N}\right) \rightarrow\left(B, g_{B}\right)$ is a 
Riemannian submersion with horizontal distribution $\mathcal{H}$, such that the circumference of each fiber $\pi^{-1}(p)$ equals $2 \pi \cdot f(p)$, and the locally well-defined $S^{1}$ action which rotates the fibers acts by isometries. The 2 -form $\omega$ on $B$ is only well defined up to sign and is realized as $\omega(X, Y)=\left\langle R^{\theta}(X, Y) W, V\right\rangle$, where $R^{\theta}$ is the curvature tensor of $\theta$, and $\{W, V\}$ is an orthonormal basis of the fiber. However, $|\omega|$ is well defined, and is all that appears in, for example, Theorem 3.1 of the next section.

\section{Conditions For POSitive And nOnNegative CURVATURE}

Now we establish necessary and sufficient conditions for an $S^{1}$-bundle to admit a metric of positive/nonnegative sectional curvature.

\section{Theorem 3.1.}

(1) If the warped connection metric on the circle bundle $S^{1} \hookrightarrow N \stackrel{\pi}{\rightarrow} B$ determined by the data $\left\{g_{B}, \theta, f\right\}$ has nonnegative (respectively positive) sectional curvature, then the following inequality is satisfied (respectively strictly satisfied) for all $p \in B$ and $X, Y \in T_{p} B$ with $X \wedge Y \neq 0$ :

$$
\left(\left(D_{X} \omega\right)(X, Y)\right)^{2} \leq\left(\left|i_{X} \omega\right|^{2}+\operatorname{hess}_{\phi}(X, X)\right) \cdot k_{B}(X, Y)
$$

where $\phi=2 f^{-2}, k_{B}(X, Y)=\langle R(X, Y) Y, X\rangle_{g_{B}}$ denotes the unnormalized sectional curvature of $\left(B, g_{B}\right)$, and $i_{X} \omega$ denotes the 1 -form on $B$ defined by $\left(i_{X} \omega\right)(Y)=\omega(X, Y)$.

(2) Conversely, if there exist structures $\left\{g_{B}, \theta, \phi\right\}$ such that the inequality is strictly satisfied for all $p \in B$ and $X, Y \in T_{p} B$ with $X \wedge Y \neq 0$, then $N$ admits a warped connection metric with positive curvature.

Part (2) of the theorem was proven in 11] by constructing a metric on the associated vector bundle in such a way that derivative considerations force the boundary of a small metric tube about the zero-section to have positive curvature. For the positively curved metric guaranteed by part (2) of the theorem, note that the function $\phi: B \rightarrow \mathbf{R}$ is not the fiber-length function.

One should not expect that structures $\left\{g_{B}, \theta, f\right\}$ satisfying the inequality nonstrictly can necessarily induce a warped connection metric of nonnegative curvature. For example, in the connection metric case ( $f$ constant) our inequality is slightly weaker than the necessary and sufficient condition in [17].

It remains to prove part (1), for which we require the following preliminary conditions for nonnegative/positive curvature:

Lemma 3.2. The warped connection metric on the oriented circle bundle $S^{1} \hookrightarrow$ $N \stackrel{\pi}{\rightarrow} B$ prescribed by the data $\left\{g_{B}, \theta, f\right\}$ has nonnegative (respectively positive) curvature if and only if the following inequalities are satisfied (respectively strictly satisfied) for all $p \in B$ and all $X, Y \in T_{p} B$ with $X \wedge Y \neq 0$ :

(1) $K_{B}(X, Y) \geq \frac{3}{4} f^{2} \omega(X, Y)^{2} \geq 0$,

(2) $f^{2}\left|i_{X} \omega\right|^{2}-\frac{4 X(X(f))}{f} \geq 0$,

(3) $\left(3 X(f) \omega(X, Y)+f\left(D_{X} \omega\right)(X, Y)\right)^{2}$

$$
\leq\left(f^{2}\left|i_{X} \omega\right|^{2}-\frac{4 X(X(f))}{f}\right) \cdot\left(K_{B}(X, Y)-\frac{3 f^{2}}{4} \omega(X, Y)^{2}\right) .
$$


Proof. The proof is essentially a generalization of Lemma 1 of [17]. Endow $N$ with the warped connection metric $g_{N}$ prescribed by the data $\left\{g_{B}, \theta, f\right\}$. Note that the three inequalities are satisfied for all $X, Y$ with $X \wedge Y \neq 0$ if and only if they are satisfied for all orthonormal $X, Y$.

Let $V$ be the unit vertical vector field on $N$ such that $f \cdot \Omega(V)=1$. For any point $p \in B$ and any 2-plane $\sigma$ in $T_{p} B$, let $X, Y$ be an orthonormal basis of $\sigma$, and let $\bar{X}, \bar{Y}$ be their horizontal lifts to $T_{\bar{p}} N$, where $\bar{p} \in \pi^{-1}(p)$. Then for any real number $\varphi$, the vectors $\{\bar{X},(\sin \varphi) \bar{Y}+(\cos \varphi) V\}$ are orthonormal and span a 2-plane $\bar{\sigma}$ in $T_{\bar{p}} N$. It is easy to see that every 2-plane in $T_{\bar{p}} N$ is spanned by orthonormal vectors of this form. The sectional curvature of $\bar{\sigma}$ is given by

$$
\begin{aligned}
K(\bar{\sigma}) & =\left(\cos ^{2} \varphi\right) K(\bar{X}, V)+\left(\sin ^{2} \varphi\right) K(\bar{X}, \bar{Y})+2(\sin \varphi \cos \varphi) R(\bar{X}, \bar{Y}, \bar{X}, V) \\
& =Q((\cos \varphi, \sin \varphi),(\cos \varphi, \sin \varphi)),
\end{aligned}
$$

where $Q$ is the quadratic form with matrix

$$
Q=\left(\begin{array}{cc}
K(\bar{X}, V) & R(\bar{X}, \bar{Y}, \bar{X}, V) \\
R(\bar{X}, \bar{Y}, \bar{X}, V) & K(\bar{X}, \bar{Y})
\end{array}\right) .
$$

By the Gray-O'Neill submersion formulas, we have

$$
K(\bar{X}, \bar{Y})=K(\sigma)-\frac{3}{4}\left\|[\bar{X}, \bar{Y}]^{v}\right\|^{2}=K(\sigma)-\frac{3}{4} f^{2} \omega(\sigma)^{2}
$$

since $[\bar{X}, \bar{Y}]=-f \omega(\sigma) V$. The bracket is computed by extending $X$ and $Y$ to locally commuting vector fields in a neighborhood of $p$ in $B$, and $\bar{X}, \bar{Y}$ are the horizontal lifts in a neighborhood of $\bar{p}$.

For the $T$-tensor of $\pi$ we have $T_{V} V=-\frac{\overline{\operatorname{grad} f}}{f}$, where $\overline{\operatorname{grad} f}$ denotes the horizontal lift of the gradient of $f$. Straightforward computations now yield

$$
\begin{aligned}
K(\bar{X}, V) & =\left\langle\left(D_{\bar{X}} T\right)_{V} V, \bar{X}\right\rangle-\left\|T_{V} \bar{X}\right\|^{2}+\left\|A_{\bar{X}} V\right\|^{2} \\
& =\left(\frac{X(f)}{f}\right)^{2}-\frac{X(X(f))}{f}-\left(\frac{X(f)}{f}\right)^{2}+\frac{f^{2}}{4}\left\|i_{X} \omega\right\|^{2} \\
& =\frac{f^{2}}{4}\left\|i_{X} \omega\right\|^{2}-\frac{X(X(f))}{f}, \\
R_{f}(\bar{X}, \bar{Y}, \bar{X}, V) & =-\frac{3}{2} X(f) \omega(\sigma)-\frac{1}{2} f \cdot\left(D_{X} \omega\right)(\sigma) .
\end{aligned}
$$

The quadratic form $Q$ is nonnegative definite (respectively positive definite) if and only if its determinant is nonnegative (respectively positive) and its two diagonal entries are nonnegative (respectively positive). This completes the proof.

Proof of part (1) of Theorem 3.1. Since the argument is local, we can assume that the bundle is orientable. Suppose first that the warped connection metric $g$ on $N$ prescribed by the data $\left\{g_{B}, \theta, f\right\}$ has nonnegative curvature. We use Cheeger's trick of rescaling $(N, g)$ along the action field of the principal $S^{1}$-action 2]. More precisely, consider the diagonal isometric $S^{1}$ action on $(N, g) \times S^{1}(r)$, where $S^{1}(r)$ denotes the circle with circumference $2 \pi r$. The quotient, $\left[(N, g) \times S^{1}(r)\right] / S^{1}=$ $(N, g) \times{ }_{S^{1}} S^{1}(r)$, is diffeomorphic to $N$, but inherits a submersion metric different from $g$. Write

$$
\left(N, g_{r}\right)=(N, g) \times S^{1} S^{1}(r) .
$$


Then $g_{r}$ will also be a metric of nonnegative curvature. In fact, $g_{r}$ is the warped connection metric prescribed by the data $\left\{g_{B}, \theta, f \cdot\left(1+(f / r)^{2}\right)^{-1 / 2}\right\}$. Therefore, the third inequality of Lemma 3.2 remains true if $f$ is replaced by

$$
f_{r}=f \cdot\left(1+(f / r)^{2}\right)^{-1 / 2}
$$

for any $r>0$. Making this substitution, then dividing both sides of the inequality by $r^{2}$ and taking the limit as $r \rightarrow 0$ produces the required inequality.

Next suppose that the warped connection metric $g$ on $N$ prescribed by the data $\left\{g_{B}, \theta, f\right\}$ has positive curvature. For $r>0,\left(N, g_{r}\right)$ has positive curvature because there are no horizontal zero-curvature planes in $N \times S^{1}(r)$. Therefore inequality (3) of Lemma 3.2 remains strictly satisfied if we replaced $f$ by $f_{r}$. However, as $r$ goes to zero, some calculations are needed to control the difference between the left and right sides of this inequality.

Let $X$ and $Y$ be vector fields on $B$. At the point $p \in B$, let $\gamma(t)$ be a geodesic with $\gamma(0)=p, \gamma^{\prime}(0)=X(p)$. Now we compute that

$$
\begin{aligned}
X\left(f_{r}\right) & =\frac{X(f) r^{3}}{\left(f^{2}+r^{2}\right)^{\frac{3}{2}}}, \\
X\left(X\left(f_{r}\right)\right) & =\frac{r^{2} X(X(f))}{\left(f^{2}+r^{2}\right)^{\frac{3}{2}}}-\frac{3 f r^{3} X(f)^{2}}{\left(f^{2}+r^{2}\right)^{\frac{3}{2}}} .
\end{aligned}
$$

Let

$$
\begin{array}{r}
H(r)=\frac{1}{r^{2}}\left[\left(f_{r}^{2}\left|i_{X} \omega\right|^{2}-\frac{4 X\left(X\left(f_{r}\right)\right)}{f_{r}}\right) \cdot\left(K_{B}(X, Y)-\frac{3 f_{r}^{2}}{4} \omega(X, Y)^{2}\right)\right. \\
\left.-\left(3 X\left(f_{r}\right) \omega(X, Y)+f_{r}\left(D_{X} \omega\right)(X, Y)\right)^{2}\right] .
\end{array}
$$

Then we can simplify $H(r)$ to be

$$
H(r)=\frac{\alpha_{1}}{f^{2}+r^{2}}+\frac{\alpha_{2}}{\left(f^{2}+r^{2}\right)^{2}}+\frac{\alpha_{3}}{\left(f^{2}+r^{2}\right)^{3}}-\frac{\beta_{1}}{f^{2}+r^{2}}-\frac{\beta_{2}}{\left(f^{2}+r^{2}\right)^{2}}-\frac{\beta_{3}}{\left(f^{2}+r^{2}\right)^{3}}
$$

where

$$
\begin{aligned}
\alpha_{1} & =\left(f^{2}\left|i_{X} \omega\right|^{2}-\frac{4 X(X(f))}{f}\right) \cdot\left(K_{B}(X, Y)-\frac{3 f^{2}}{4} \omega(X, Y)^{2}\right), \\
\alpha_{2} & =12 X(f)^{2}\left(K_{B}(X, Y)-\frac{3 f^{2}}{4} \omega(X, Y)^{2}\right) \\
& +\frac{3 f^{4}}{4} \omega(X, Y)^{2}\left(f^{2}\left|i_{X} \omega\right|^{2}-\frac{4 X(X(f))}{f}\right), \\
\alpha_{3} & =9 f^{4} X(f)^{2} \omega(X, Y)^{2} \\
\beta_{1} & =\left(3 X(f) \omega(X, Y)+f\left(D_{X} \omega\right)(X, Y)\right)^{2} \\
\beta_{2} & =6 f^{2} X(f) \omega(X, Y) \sqrt{\beta_{1}} \\
\beta_{3} & =\alpha_{3} .
\end{aligned}
$$


Thus $H(r)=\frac{\alpha_{1}-\beta_{1}}{f^{2}+r^{2}}+\frac{\alpha_{2}-\beta_{2}}{\left(f^{2}+r^{2}\right)^{2}}$ as a function of $r$. Note that by Lemma 3.2 we have $\alpha_{1}-\beta_{1}>0$. Now consider

$$
\begin{aligned}
0 & \leq\left(2 \sqrt{3} \sqrt{K_{B}(X, Y)-\frac{3 f^{2}}{4} \omega(X, Y)^{2}}-\frac{\sqrt{3}}{2} f^{2} \omega(X, Y) \sqrt{f^{2}\left|i_{X} \omega\right|^{2}-\frac{4 X(X(f))}{f}}\right)^{2} \\
& =\alpha_{2}-6 X(f) f^{2} \omega(X, Y) \sqrt{K_{B}(X, Y)-\frac{3 f^{2}}{4} \omega(X, Y)^{2}} \cdot \sqrt{f^{2}\left|i_{X} \omega\right|^{2}-\frac{4 X(X(f))}{f}} \\
& <\alpha_{2}-\beta_{2},
\end{aligned}
$$

i.e., $\alpha_{2}-\beta_{2}>0$. Hence, $H(r)$ is positive valued for all $r$. The derivative of the function $H(r)$ is $H^{\prime}(r)=-2 r\left(\frac{\alpha_{1}-\beta_{1}}{f^{2}+r^{2}}-\frac{2\left(\alpha_{2}-\beta_{2}\right)}{\left(f^{2}+r^{2}\right)^{2}}\right)$, which by the above remarks is always negative for all positive values of $r$. Hence, $H(r)$ is a decreasing function. Therefore $\lim _{r \rightarrow 0+} H(r)$ is positive. This yields the required inequality.

We now explore some consequences of our results.

(i) Suppose that the circle bundle $S^{1} \hookrightarrow N \stackrel{\pi}{\rightarrow} B$ has a connection metric such that all 2-planes at some point $\bar{p} \in N$ have positive curvature. Then $\left|i_{X} \omega\right|>0$ for all $X \in T_{p} B$, where $p=\pi(\bar{p})$. Hence $\omega$ is nondegenerate at $p$, so $B$ is evendimensional and $N$ odd-dimensional.

(ii) Suppose that the circle bundle $S^{1} \hookrightarrow N \stackrel{\pi}{\rightarrow} B$ has a warped connection metric with positive curvature. Then at a local minimum $p \in B$ of the fiber-length function $f$, we have that $\left|i_{X} \omega\right|>0$ for all $X \in T_{p} B$. Hence $\omega$ is nondegenerate at $p$, so $B$ is even-dimensional and $N$ odd-dimensional. This also follows from Berger's result on the vanishing of Killing fields on even-dimensional manifolds of positive curvature.

Along these lines we present an interesting question. Recall that a manifold is said to be almost positively curved if every 2-plane at almost every point has positive curvature. Recently B. Wilking produced many interesting examples of almost positively curved manifolds in [16], including examples that violate Synge's theorem for positively curved manifolds. It is natural to ask whether Berger's result extends to this intermediate class between positive curvature and nonnegative curvature.

Question 3.3. Does every Killing field on an even-dimensional, almost positively curved manifold vanish somewhere?

\section{Rigidity In EVEn DIMENSIONS}

If $B$ is odd-dimensional, it is possible for a circle bundle over $B$ to admit an invariant metric with nonnegative (but not positive) curvature. The principal fibration $S^{1} \hookrightarrow \mathrm{SU}(3) \rightarrow N_{p, q}$ which yields the homogeneous Aloff-Wallach spaces (see section 7) is an example. However, we have the following rigidity result. We present two proofs, as the techniques used in either proof may be of independent interest.

Proposition 4.1. For a nonnegatively curved warped connection metric $g_{N}$ on a circle bundle $S^{1} \hookrightarrow N \stackrel{\pi}{\rightarrow} B^{2 n+1}$, any locally minimal length fiber is contained in a flat, totally geodesic, immersed cylinder. In particular, the local minima of the fiber-length function cannot be isolated. 
First proof of Proposition 4.1. Let $p \in B$ be a minimum for the fiber-length function $f$. The fiber $\pi^{-1}(p)$ is totally geodesic. Let $\rho_{t}$ parametrize the $S^{1}$ action along this fiber (even if the bundle is nonorientable, $N$ admits an isometric $S^{1}$-action over a neighborhood of $p$ in $B$ ). Let $\tau_{t}$ be the parallel transport map along this (geodesic) fiber. Note that $\pi_{*}=\pi_{*} \circ\left(\rho_{t}\right)_{*}$.

Let $\bar{p} \in \pi^{-1}(p)$. Now consider the maps, $g_{t}:=\tau_{t}^{-1} \circ\left(\rho_{t}\right)_{*}: T_{\bar{p}} N \rightarrow T_{\bar{p}} N$, defined for all $t$. We claim that this generates a one-parameter subgroup in the orthogonal group of isometries of $T_{\bar{p}} N$. To show that $\left\{g_{t}\right\}$ is a one-parameter subgroup, we need to show that $g_{t} \circ g_{s}=g_{s} \circ g_{t}$. Let $\mathcal{H}$ denote the horizontal distribution along the fiber. Then we may view $g_{t}$ as a map from $\mathcal{H}$ to itself, since $g_{t}$ is an isometry that preserves vectors along the fiber. Now the commutativity of the $g_{t}$ is clear since we require that on $\mathcal{H}$ we have $\left(\rho_{t}\right)_{*} \circ \tau_{s}^{-1}=\left(\rho_{s}\right)_{*} \circ \tau_{t}^{-1}$ which is turn follows from the general fact that isometries preserve parallel vector fields. Hence, $g_{t}=\exp (t X)$ for some skew symmetric endomorphism $X$.

$X$ is a skew symmetric endomorphism on an odd-dimensional space (the complement to the fiber direction at $\bar{p}$ ). Since its eigenvalues come in purely imaginary pairs, it must have a zero eigenvalue. Then $\exp (t X)$ has eigenvalue 1 for all $t$, and a fixed vector corresponding to this eigenvalue, say $v$. This implies that for all $t$, the parallel transport of $v$ agrees with the circle action. So, the image of $v$ under $\left(\rho_{t}\right)_{*}$ in $\mathcal{H}$ is an invariant parallel field, and we may look at its exponential image which is a cylinder. The image of the fiber $\pi^{-1}(p)$ along $v$ is also a fiber, since $\left(\rho_{t}\right)_{*}(v)$ was constructed to be an invariant parallel field. By Rauch's second comparison theorem, it follows that these nearby fibers must have the same length and are therefore also geodesics. From the equality discussion of Rauch's theorem, the resulting cylinder is flat and totally geodesic.

Second proof of Proposition 4.1. Let $p \in B$ be a local minimum of the fiber-length function $f: B \rightarrow \mathbf{R}^{+}$. Since $B$ is odd-dimensional, there exists a vector $X \in T_{p} B$ such that $\left|i_{X} \omega\right|=0$. Let $\bar{X}$ denote the horizontal lift of $X$ to a point $\bar{p} \in F=$ $\pi^{-1}(p)$. Let $\alpha(t)$ be the geodesic on $B$ with $\alpha^{\prime}(0)=X$, and let $\bar{\alpha}(t)$ denote its lift to a horizontal geodesic in $N$ with $\bar{\alpha}(0)=\bar{p}$.

Let $J(t)$ be a holonomy Jacobi field along $\bar{\alpha}$, i.e., $J(t)$ is the variational field for the family of horizontal lifts of $\alpha$ to all points of the fiber $F$. Choose $J(0)$ to be the vertical vector whose norm equals $f(p)=$ the length of the fiber $F$. In general for holonomy Jacobi fields

$$
J^{\prime}(0)=A_{\bar{\alpha}^{\prime}(0)} J(0)+T_{J(0)} \bar{\alpha}^{\prime}(0) .
$$

In this case, the $T$-tensor term vanishes because any minimal-length fiber is totally geodesic and the $A$-tensor term vanishes because $i_{X} \omega=0$. So $J^{\prime}(0)=0$.

Using the terminology of [14], $J(t)$ is a "strong $F$-Jacobi field", meaning that it is the variational field for a family of geodesics which begin at points of $F$ and are all orthogonal to $F$, and that $J^{\prime}(0)$ is tangent to $F$. We can therefore apply Rauch's comparison Theorem for submanifolds. Thus there exists $t_{0}>0$ such that for $t \in\left[0, t_{0}\right],|J(t)| \leq|\tilde{J}(t)|=|J(0)|$ (the comparison field $\tilde{J}(t)$ is in flat space and has $\tilde{J}^{\prime}(0)=0$, so it has constant norm).

But by construction, $|J(t)|=f(\alpha(t))$, and since $F$ was assumed to be a locally minimal-length fiber, $|J(t)|$ must be constant for $t \in\left[0, t_{0}\right]$. By the equality discussion of Rauch's theorem, $\operatorname{span}\left\{J(t), \alpha^{\prime}(t)\right\}$ is a zero-curvature two-plane for all 
$t \in\left[0, t_{0}\right]$, so $\left|I_{\alpha^{\prime}(t)} \omega\right|=0$. It follows that the cylinder $\pi^{-1}\left(\alpha\left(\left[0, t_{0}\right]\right)\right)$ is flat and totally geodesic.

Since $t_{0}$ depends only on an upper curvature bound for $N$, we can repeatedly re-apply this argument to extend the interval $\left[0, t_{0}\right]$ to $[0, \infty)$. By replacing $X$ with $-X$ we can extend this interval to $(-\infty, \infty)$.

Remark 4.2. One can prove further rigidity in the case that the totally geodesic flat cylinder $\pi^{-1}(\alpha(-\infty, \infty))$ has self-intersections. Suppose the geodesic $\alpha$ in $B$ has a self-intersection point $p$, so that $\alpha\left(t_{1}\right)=\alpha\left(t_{2}\right)$. Then $\left|i_{X} \omega\right|=0$ for all $X \in \operatorname{span}\left\{\alpha^{\prime}\left(t_{1}\right), \alpha^{\prime}\left(t_{2}\right)\right\}$. Since the null-space of $\omega$ at $p$ is always odd-dimensional, we in fact have a three-dimensional space $W \subset T_{p} B$ so that any geodesic in $B$ tangent to a vector in $W$ lifts to a totally geodesic flat cylinder in $N$.

\section{TRIVIAL CIRCLE BUNDLES}

In this section, we explore the rigidity of nonnegatively curved warped connection metrics on trivial circle bundles. We begin by mentioning that the following theorem, which follows from a result in [12], can also be proven easily using our inequality:

Proposition 5.1. Any connection metric with nonnegative curvature on the trivial bundle $S^{1} \hookrightarrow S^{2} \times S^{1} \rightarrow S^{2}$ is a product metric.

Proof. Define $f: S^{2} \rightarrow \mathbf{R}$ so that $\omega=f \cdot \operatorname{dvol}$. Then $\int_{S^{2}} f \mathrm{~d} v o l=0$ (the Euler class of the bundle). So there exists a point $p \in S^{2}$ with $f(p)=0$. Along any geodesic, $\gamma(t)$, in $S^{2}$ with $\gamma(0)=p$, the inequality of Theorem 3.1 reads:

$$
f^{\prime}(t)^{2} \leq k(t) \cdot f(t)^{2}
$$

where $f(t)=f(\gamma(t))$ and $k(t)$ is the Gauss curvature at $\gamma(t)$. Since $f(0)=0$, it follows that $f(t)=0$ for all $t$, so $\omega \equiv 0$.

The above proof does not work if $S^{2}$ is replaced with a higher-dimensional manifold. In fact, we have the following proposition.

Proposition 5.2. There exists a nonnegatively curved connection metric on the trivial bundle $S^{1} \hookrightarrow S^{1} \times S^{3} \rightarrow S^{3}$ which is not a product metric.

Proof. Let $g$ denote the unit-round metric on $S^{3}$, and let $\mathbf{R}$ act isometrically on $\left(S^{3}, g\right)$ by flow along the Hopf vector field $X$. Consider the natural diagonal action by isometries of $\mathbf{R}$ on $\left(S^{3}, g\right) \times \mathbf{R}$. We have

$$
\left(S^{3}, g\right) \times \mathbf{R} \rightarrow\left[\left(S^{3}, g\right) \times \mathbf{R}\right] / \mathbf{Z} \stackrel{\pi}{\rightarrow}\left[\left(S^{3}, g\right) \times \mathbf{R}\right] / \mathbf{R} .
$$

The second map, $\pi$, is a Riemannian submersion from $\left[\left(S^{3}, g\right) \times \mathbf{R}\right] / \mathbf{Z}$, which is diffeomorphic to $S^{3} \times S^{1}$, to $\left[\left(S^{3}, g\right) \times \mathbf{R}\right] / \mathbf{R}$, which is diffeomorphic to $S^{3}$. Because $X$ has constant length, it is straightforward to verify that the fibers of $\pi$ all have the same circumference, and so are totally geodesic. However, it can also be checked that the metric is not a product metric. The calculations here are analogous to Claims 3.1 and 3.2 of [7].

In the above construction, $\left\{\left(S^{3}, g\right), X\right\}$ could be replaced by a Killing field on any nonnegatively curved compact Riemannian manifold $B$. The result is a warped connection metric of nonnegative curvature on the trivial $S^{1}$ bundle over $B$, which is a connection metric if and only if the Killing field has constant length. All 
known examples of nonnegatively curved warped connection metrics on trivial circle bundles come from this construction; see [7].

\section{An example: The Aloff-Wallach spaces}

Since the Aloff-Wallach spaces and the Eschenburg spaces which fibre over the twisted flag are the only known examples of positively curved warped connection metrics on circle bundles, it is perhaps worth understanding explicitly the inequality of Theorem 3.1 for these spaces. In this section, we summarize the result one gets for the Aloff-Wallach spaces. We begin with a brief review of the construction of these spaces. Consider the following nested subgroups of $\mathrm{SU}(3)$ :

$$
\mathrm{S}_{p, q}^{1} \subset \mathrm{T}^{2} \subset \mathrm{U}(2) \subset \mathrm{SU}(3)
$$

with Lie algebras

$$
\mathfrak{s}_{p, q}^{1} \subset \mathfrak{t}^{2} \subset \mathfrak{u}(2) \subset \mathfrak{g}
$$

where $\mathrm{S}_{p, q}^{1}=\left\{\operatorname{diag}\left(z^{p}, z^{q}, z^{-(p+q)}\right) \mid z \in \mathrm{U}(1)\right\}$, the integers $p$ and $q$ are relatively prime, $\mathrm{T}^{2}$ is the maximal torus of $\mathrm{SU}(3)$ containing $\mathrm{S}_{p, q}^{1}$, and $\mathrm{U}(2) \subset \mathrm{SU}(3)$ is the embedding $\left\{\left(\begin{array}{cc}A & 0 \\ 0 & (\operatorname{det} A)^{-1}\end{array}\right) \mid A \in U(2)\right\}$.

The Aloff-Wallach space, $N_{p, q}$, is defined as the quotient $\mathrm{SU}(3) / \mathrm{S}_{p, q}^{1}$. According to [1], $N_{p, q}$ admits a one-parameter family of homogeneous metrics of positive curvature as long as $0 \notin\{p, q, p+q\}$.

In fact, if $0 \notin\{p, q, p+q\}$, then after possibly altering $\{p, q\}$ so that the new circle is conjugate to the original in $S U(3)$, the following metric on $N_{p, q}$ will be positively curved for properly chosen $\lambda$. Let $g_{0}$ denote the bi-invariant metric on $\mathrm{SU}(3)$ determined by the equation

$$
\langle X, Y\rangle_{g_{0}}=\operatorname{Re}\left(\operatorname{trace}\left(X Y^{*}\right)\right) .
$$

Let $g$ denote the left-invariant $\mathrm{Ad}_{\mathrm{U}(2)}$-invariant metric on $\mathrm{SU}(3)$ obtained from $g_{0}$ by rescaling in the direction of $\mathfrak{u}(2)$ by some positive real number $\lambda$ as follows:

$$
\left\langle X_{1}+X_{2}, Y_{1}+Y_{2}\right\rangle_{g}=\lambda\left\langle X_{1}, Y_{1}\right\rangle_{g_{0}}+\left\langle X_{2}, Y_{2}\right\rangle_{g_{0}}
$$

where $X_{1}, Y_{1} \in \mathfrak{u}(2)$ and $X_{2}, Y_{2} \perp \mathfrak{u}(2)$. Choosing the quotient metrics on $\mathrm{SU}(3) / \mathrm{S}_{p, q}^{1}$ and $\mathrm{SU}(3) / \mathrm{T}^{2}$, we have the following chain of Riemannian submersions:

$$
\begin{aligned}
& (\mathrm{SU}(3), g) \stackrel{f}{\rightarrow}\left(N_{p, q}=\mathrm{SU}(3) / \mathrm{S}_{p, q}^{1}\right) \stackrel{\pi}{\rightarrow}\left(F^{6}=\mathrm{SU}(3) / \mathrm{T}^{2}\right) \rightarrow\left(\mathbf{C P}^{2}=\mathrm{SU}(3) / \mathrm{U}(2)\right) . \\
& \begin{aligned}
\text { Let } p=\pi \circ f . \text { Choose } \\
\qquad, Y \quad \mathfrak{g} \ominus \mathfrak{t}^{2}=\left\{v \in \mathfrak{g} \mid v \perp \mathfrak{t}^{2}\right\} \\
\quad=\left(\mathfrak{u}(2) \ominus \mathfrak{t}^{2}\right) \oplus(\mathfrak{g} \ominus \mathfrak{u}(2)) \\
\quad=\left\{\left(\begin{array}{ccc}
0 & z_{0} & 0 \\
-\bar{z}_{0} & 0 & 0 \\
0 & 0 & 0
\end{array}\right) \mid z_{0} \in \mathbf{C}\right\} \oplus\left\{\left(\begin{array}{ccc}
0 & 0 & z_{1} \\
0 & 0 & z_{2} \\
-\bar{z}_{1} & -\bar{z}_{2} & 0
\end{array}\right) \mid z_{1}, z_{2} \in \mathbf{C}\right\} .
\end{aligned}
\end{aligned}
$$

We may write

$$
X=\left(\begin{array}{ccc}
0 & z_{0} & z_{1} \\
-\bar{z}_{0} & 0 & z_{2} \\
-\bar{z}_{1} & -\bar{z}_{2} & 0
\end{array}\right) \text { and } Y=\left(\begin{array}{ccc}
0 & w_{0} & w_{1} \\
-\bar{w}_{0} & 0 & w_{2} \\
-\bar{w}_{1} & -\bar{w}_{2} & 0
\end{array}\right)
$$

Slightly tedious calculations then yield the following proposition. 


\section{Proposition 6.1.}

$$
\begin{aligned}
\omega\left(p_{*} X, p_{*} Y\right)= & -2 \cdot \operatorname{Im}\left((p+q) \bar{z}_{0} w_{0}+q \bar{z}_{1} w_{1}-p \bar{z}_{2} w_{2}\right) \\
D_{\left(p_{*} X\right)} \omega\left(p_{*} X, p_{*} Y\right)=\operatorname{Im}[ & -((3-2 \lambda) q+(3-\lambda) p) z_{0} z_{2} \bar{w}_{1} \\
& \left.+((3-2 \lambda) p+(3-\lambda) q) z_{0} \bar{z}_{1} w_{2}+\lambda(p-q) \bar{z}_{1} z_{2} w_{0}\right] .
\end{aligned}
$$

From the first formula we see that $\omega$ is nondegenerate if and only if $0 \notin\{p, q$, $p+q\}$, which is exactly the sufficient condition in [1] for $N_{p, q}$ to admit an invariant metric of positive curvature. This fact is also mentioned in [18, along with a proof that the excluded circle bundle $N_{1,0}$ does not admit a connection whose curvature form $\omega$ is nondegenerate. A connection in a circle bundle is called fat if $\omega$ is nondegenerate. Fatness is equivalent to the positivity of the vertizontal curvatures. Hence, $N_{1,0}$ does not admit a connection metric of positive curvature.

Remark 6.2. Using the terminology of [10], we call a connection $\theta$ in a principal $S^{1}$ bundle radially symmetric if for all $p \in B$ and all $X, Y \in T_{p} B,\left(D_{X} \omega\right)(X, Y)=0$. It follows from Lemma 3.2 or from the main result of [10] that if $B$ admits a metric of positive curvature, then any radially-symmetric connection in any circle bundle over $B$ induces a connection metric of nonnegative curvature. However, if a connection in a circle bundle is radially symmetric, then it is in fact parallel, i.e., $D \omega=0$. To see this, note that since $\left(D_{X+Z} \omega\right)(X+Z, Y)=0$, it follows that $\left(D_{X} \omega\right)(Y, Z)=-\left(D_{Y} \omega\right)(X, Z)$. Thus, $(X, Y, Z) \mapsto\left(D_{X} \omega\right)(Y, Z)$ is alternating. Next use that fact that $\omega$ is closed, and that the exterior derivative of $\omega$ is the skew-symmetrization of its covariant derivative:

$0=(d \omega)(X, Y, Z)=\left(D_{X} \omega\right)(Y, Z)-\left(D_{Y} \omega\right)(X, Z)+\left(D_{Z} \omega\right)(X, Y)=3\left(D_{X} \omega\right)(Y, Z)$.

If $B$ has positive curvature, then Theorem 3.1 implies that a radially-symmetric connection $\theta$ induces a connection metric of positive curvature if and only if $\theta$ is fat, i.e., if and only if $\omega$ is a Kähler form. Therefore, $B$ is a Kähler manifold of positive curvature. The only Kähler manifolds with positive curvature are the complex projective spaces $\mathbf{C P}^{n}$ (with $N$ being the standard sphere or any lens space) [9].

\section{REFERENCES}

1. S. Aloff and N. Wallach, An infinite family of distinct 7-manifolds admitting positively curved Riemannian structures, Bull. Amer. Math. Soc. 81 (1975) 93-97. MR0370624 (51:6851)

2. J. Cheeger, Some examples of manifolds of nonnegative curvature, J. Differential Geom. 8 (1972), 623-628. MR0341334(49:6085)

3. J.-H. Eschenburg, Freie isometrische Aktionen auf kompakten Lie-Gruppen mit positiv gekrümmten Orbiträumen, Schriftenreihe des Mathematischen Instituts der Universität Münster (2) 32, Universität Münster, Mathematisches Institut, Münster, 1984. MR0758252 (86a:53045)

4. J.-H. Eschenburg, Inhomogeneous spaces of positive curvature, Differential Geom. Appl. 2 (1992), 123-132. MR.1245552 (94j:53044)

5. K. Fukaya, T. Yamaguchi, The fundamental groups of almost non-negatively curved manifolds, Ann. of Math. (2) 136 (1992), 253-333. MR1185120 (93h:53041)

6. P. Gilkey, J.H. Park, W. Tuschmann, Invariant metrics of positive Ricci curvature on principal bundles, Math. Z. 227 (1998), 455-463. MR1612669 (2000b:53042)

7. D. Gromoll and K. Tapp, Nonnegatively curved metrics on $S^{2} \times \mathbf{R}^{2}$, preprint.

8. J. C. Nash, Positive Ricci curvature on fiber bundles, J. Diff. Geom. 14 (1979), 241-265. MR0587552 (81k:53039)

9. Y. T. Siu and S. T. Yau, Compact Kahler manifolds of positive bisectional curvature, Invent. Math. 59 (1980), 189-204. MR0577360 (81h:58029) 
10. M. Strake and G. Walschap, Connection metrics of nonnegative curvature on vector bundles, Manuscripta Math. 66 (1990), 309-318. MR1031200 (91a:53070)

11. K. Tapp, Conditions for nonnegative curvature on vector bundles and sphere bundles, Duke Math J. 116 (2003), No. 1, 77-101. MR1950480 (2004b:53045)

12. G. Walschap, Nonnegatively curved manifolds with souls of dimension 2, J. Differential Geom. 27 (1988), 525-537. MR0940117 (89g:53067)

13. G. Walschap and M. Özaydin, Vector bundles with no soul, Proc. Amer. Math. Soc. 120 (1994), no. 2, 565-567. MR 1162091 (94d:53057)

14. F. Warner, Extension of the Rauch comparison Theorem to submanifolds, Trans. A.M.S. 122 (1966), 341-356. MR0200873 (34:759)

15. A. Weinstein, Fat bundles and symplectic manifolds, Adv. in Math. 37 (1980), 239-250. MR0591728 (82a:53038)

16. B. Wilking, Manifolds with positive sectional curvature almost everywhere, Invent. Math. 148 (2002), 117-141. MR1892845 (2003a:53049)

17. D. Yang, On complete metrics of nonnegative curvature on 2-plane bundles, Pacific J. of Math., Vol. 171, No. 2, 1995. MR1372245 (96k:53034)

18. W. Ziller, Fatness Revisited, lecture notes, 1999.

Department of Mathematics, University of Oklahoma, Norman, Oklahoma 73019

E-mail address: shankar@math.ou.edu

Department of Mathematics, Bryn Mawr College, Philadelphia, Pennsylvania 19010

E-mail address: ktapp@brynmawr.edu

Mathematisches Institut, Westfälische Wilhelms-Universität Münster, EinsteinStrasse 62, D-48149 MÜnster, Germany

E-mail address: wtusch@math.uni-muenster.de 